University of Nebraska - Lincoln

DigitalCommons@University of Nebraska - Lincoln

December 1984

\title{
YELLOW GIANTS IN YOUNG CLUSTERS. II. A COMPARISON OF OBSERVATION WITH THEORY
}

\author{
Edward G. Schmidt \\ University of Nebraska-Lincoln, eschmidt1@unl.edu
}

Follow this and additional works at: https://digitalcommons.unl.edu/physicsschmidt

Part of the Physics Commons

Schmidt, Edward G., "YELLOW GIANTS IN YOUNG CLUSTERS. II. A COMPARISON OF OBSERVATION WITH THEORY" (1984). Edward Schmidt Publications. 32.

https://digitalcommons.unl.edu/physicsschmidt/32

This Article is brought to you for free and open access by the Research Papers in Physics and Astronomy at DigitalCommons@University of Nebraska - Lincoln. It has been accepted for inclusion in Edward Schmidt Publications by an authorized administrator of DigitalCommons@University of Nebraska - Lincoln. 
The ASTROPHYSICAL JoURNAL, 287:261-267, 1984 December 1

C 1984. The American Astronomical Society. All rights reserved. Printed in U.S.A.

\title{
YELLOW GIANTS IN YOUNG CLUSTERS. II. A COMPARISON OF OBSERVATION WITH THEORY
}

\author{
EDWARD G. SCHMIDT ${ }^{1}$ \\ Department of Physics and Astronomy, University of Nebraska \\ Received 1984 March 30; accepted 1984 June 14
}

\begin{abstract}
Luminosities and effective temperatures are calculated for 17 nonvariable yellow giants and supergiants and nine Cepheids which were previously identified as members of open clusters. These stars are identified as being in the helium core burning stage of evolution. A comparison with the predictions of pulsation theory does not show any disagreement. On the other hand, several comparisons with the theory of stellar evolution produce discrepancies which suggest that effects neglected in the calculations may play an important role in the evolution of these stars.

Subject headings: clusters: open — stars: Cepheids — stars: evolution - stars: supergiants
\end{abstract}

\section{INTRODUCTION}

The yellow giants and supergiants are of interest both in connection with the theory of stellar evolution and the theory of stellar pulsation. Most of these stars are in the helium core burning stage and have evolved back into the yellow giant region from the red giant branch. Their location in the H-R diagram thus constrains post-main-sequence evolutionary models. Furthermore, in this region there is a mass-luminosity relation, while nearer to the Hayashi limit we would expect more mixing of masses at a given luminosity. Thus, comparisons with theory are less ambiguous for these stars. Since Cepheid variables are of this type, the yellow giants are also of interest in connection with the location of the instability region.

It is difficult to obtain accurate luminosities, intrinsic colors, temperatures, and ages for field yellow giants. Those in open clusters are therefore of special interest. Unfortunately, the number of such stars for which cluster membership is well established is relatively small, and many dubious cases exist. To improve this situation a survey was undertaken to locate likely cluster members. The results were discussed in Paper I (Schmidt 1984a). In that paper 22 likely cluster yellow giants (20 of which are nonvariables) were identified. These stars; together with Cepheids known to be cluster members, are used in the present paper to discuss several questions of interest to stellar evolution and pulsation. These include the questions of what fraction of stars in the Cepheid instability strip are nonvariables, the location of the edges of the instability strip, the distribution of stars in the yellow giant region as compared with the predictions of theory, the location of helium core burning stars in the H-R diagram with respect to core hydrogen burning stars of the same age, and the typical age spread of stars in individual clusters.

\section{LUMINOSITIES AND EFFECTIVE TEMPERATURES}

In Paper I we identified 22 stars which are likely members of clusters. Two of these are variables. Star 7400P in NGC 6067 is

\footnotetext{
1 Visiting Astronomer at Kitt Peak National Observatory and Cerro Tololo Inter-American Observatory which are operated by the Association for Research in Astronomy, Inc., under contract from the National Science Foundation.
}

a Cepheid with a period of 11.5 days (Eggen 1983a), while HR 4511 in Stock 14 is either a long-period Cepheid or a Cepheidlike variable (Eggen 1983b). Additionally, NGC 752 is older than is appropriate to this sample, and this cluster is omitted from the present discussion. The 17 remaining yellow giants are listed in Table 1.

Because many of the clusters in Table 1 are in crowded regions and have variable extinction, the distance moduli and color excesses are difficult to determine. The four-color and $\mathrm{H} \beta$ photometric system is useful in such cases because it is capable of producing accurate color excesses and distance moduli for individual B stars. There is only one cluster in Table 1, NGC 2546, which has not been studied in that system. The last column of the table contains references to the photometry. In each case the B stars in the cluster were used to determine the distance modulus and color excess from the calibration of Crawford (1978). These are given in the third and fourth columns. When there is appreciable variation of the extinction over the cluster face, the best value for the yellow giant was found by interpolating among nearby stars. In such cases there is an estimate of the error involved following the color excess. The absence of an estimated error indicates the the extinction is constant and the error should be less than $0.01 \mathrm{mag}$ in $E(b-y)$. For NGC 2546 we have used the distance modulus (corrected to a Hyades distance modulus of 3.3) and color excess obtained by Becker and Fenkhart (1971) from broadband photometry.

In Table 2 we list a number of clusters containing Cepheid variables. These clusters were used to calibrate the periodluminosity-color relation (Schmidt 1984b). This list does not contain all those Cepheids which have been suggested for cluster membership. However, it does include those with reasonably well-established membership and is likely to be as complete as the present nonvariable yellow giant list. All of these clusters have been observed in the four-color and $\mathrm{H} \beta$ system, and the distance moduli and color excesses from Schmidt (1984b) were used. They are consistent with those in Table 1.

For each star in Tables 1 and 2 we list the absolute magnitude and several intrinsic colors. Most of the photometric data for Table 1 comes from Paper I. When this is not the case the footnotes give the sources of the colors and magnitudes. 
TABLE 1

Yellow Giants in Clusters.

\begin{tabular}{|c|c|c|c|c|c|c|c|c|c|c|c|}
\hline Cluster & Star & $V_{0}-N_{v}$ & $E(b-y)$ & $M_{v}$ & $(b-y)_{0}$ & $(B-V)_{0}$ & $(V-R)_{0}$ & $(R-I)_{0}$ & $\log L / L_{\odot}$ & $\log T_{\text {eff }}$ & References \\
\hline NGC $2168 \ldots \ldots$ & 101 & 9.5 & 0.194 & -1.8 & 0.51 & 0.91 & 0.64 & 0.41 & 2.69 & 3.740 & 2 \\
\hline \multirow[t]{3}{*}{ NGC $2287 \ldots \ldots$} & f & 1 & 1 & -1.6 & 0.66 & 1.11 & $\ldots$ & 0.54 & 2.63 & 3.689 & 3 \\
\hline & 75 & 9.2 & 0.032 & $-1 . .9$ & 0.71 & 1.18 & $\ldots$ & 0.58 & 2.76 & 3.676 & \\
\hline & 97 & 1 & & -1.7 & 0.67 & 1.11 & $\ldots$ & 0.53 & 2.67 & 3.690 & \\
\hline \multirow{2}{*}{ NGC 2546} & 99 & & 014. & -2.3 & 0.61 & 1.02 & $\ldots$ & $\ldots$ & 2.90 & 3.708 & 4 \\
\hline & 356 & 9.9: & $0.14:\}$ & -2.3 & 0.64 & 1.15 & & & 2.92 & 3.692 & \\
\hline \multirow{2}{*}{ NGC 3114} & 76 & $\{10.3$ & 0.060 & -2.1 & 0.72 & 1.17 & 0.91 & 0.53 & 2.84 & 3.678 & \\
\hline & 159 & & & -2.1 & 0.66 & 1.12 & 0.84 & 0.50 & 2.83 & 3.692 & \\
\hline NGC 6067 & $7400 \mathrm{f}$ & 10.4 & $0.23 \pm 0.03$ & -2.4 & 0.12 & 0.20 & 0.26 & 0.11 & 2.84 & 3.858 & 6 \\
\hline NGC 6475 & 58 & 7.1 & $0.06 \pm 0.01$ & -1.8 & 0.61 & 1.00 & & & 2.70 & 3.709 & 7 \\
\hline M25 ............. & 150 & 8.8 & $0.36 \pm 0.03$ & -3.0 & 0.68 & 1.19 & 0.97 & 0.55 & 3.20 & 3.674 & 8 \\
\hline NGC $6664 \ldots \ldots$. & 29 & 10.7 & $0.65 \pm 0.05$ & -2.8 & & 0.98 & 0.86 & 0.44 & 3.10 & 3.709 & 9 \\
\hline NGC $7654 \ldots \ldots$ & 2 & 10.8 & $0.45 \pm 0.05$ & -4.5 & 0.26 & 0.63 & 0.53 & 0.29 & 3.73 & 3.791 & 10 \\
\hline
\end{tabular}

References.-(1) Schmidt 1980. (2) Forbes and Schmidt 1984. (3) Eggen 1981; $(R-I)$ from Schmidt 1976. (4) Becker and Fenkart 1971 from broad-band photometry. (5) Schmidt 1982a. (6) Eggen 1983a. (7) Snowden 1976 and Forbes and Schmidt 1984. (8) Schmidt 1982b. (9) Schmidt 1982c variable color excess required extrapolation. (10) Danford and Thomas $1981 ;(b-y)$ from this source also.

For Table 2 we have taken the absolute magnitudes and $\langle B\rangle_{0}-\langle V\rangle_{0}$ colors from Schmidt $(1984 b)$. The sources of the other colors are given in the footnotes.

In calculating the absolute magnitudes we have used $A=4.28 E(b-y)$ (Crawford and Mandewewala 1976). The color excesses in $(b-y)$ are not dependent on the color of the star because the bands are relatively narrow. Thus, the color excesses obtained from the cluster B stars can be used directly. They were converted to $(B-V)$ using $E(b-y)=0.777$ $E(B-V)$ (Crawford and Mandewewala 1976) which is valid for B stars. They were then corrected for the color of the star following Fernie (1963) before being used to correct the colors of the yellow giants and Cepheids. For $(V-R)$ and $(R-I)$ no studies have been made of the effects of the band width on the color excesses. Since the bands are comparable in width to the $\mathrm{B}$ and $\mathrm{V}$ bands, we have assumed that the effects are similar and have converted the color excesses using $E(V-R)=$ $0.86 E(B-V)$ and $E(R-I)=0.82 E(B-V)$.

Tables 1 and 2 also list the luminosities and effective temperatures of the stars. The luminosities were calculated using the bolometric corrections of Sandage and Gratton (1963). In determining the temperature for each star we have used all available color data. Pel (1978) has derived a scale appropriate to stars in and near the Cepheid region of the H-R diagram.
We have used this scale in the form

$$
\log T_{\text {eff }}=3.886-0.175(B-V)
$$

which was given by Cox (1979). For $(b-y)$ we derived a relation between $(B-V)$ and $(b-y)$ from all the dereddened colors in Tables 1 and 2 and used it to transform equation 1 into the form

$$
\log T_{\text {eff }}=3.883-0.288(b-y) .
$$

For $(V-R)$ and $(R-I)$ we have used the temperatures given by Johnson (1966). The values from this scale average 0.028 and 0.023 cooler in the logarithm than those from equations (1) and (2) so they were corrected accordingly before all available estimates were averaged for each star. For the most part, the various colors gave temperatures consistent at the level of 0.01 in the logarithm although a few obviously discrepant values were deleted. The mean temperatures in Tables 1 and 2 should be consistent with each other at that level.

The effective temperature scale of Pel is only one of several suggested for stars of this type (see Pel 1980 for a summary). It is adopted here because it is one of the more recent and falls near the middle of the range of the various scales. The others deviate by up to \pm 0.02 in $\log T_{\text {eff }}$ for stars at about the tem-

TABLE 2

\begin{tabular}{|c|c|c|c|c|c|c|c|c|c|c|}
\hline Cepheid & Period & Cluster & $\left\langle M_{v}\right\rangle$ & $\langle b-y\rangle_{0}$ & $\langle B\rangle_{0}-\langle V\rangle_{0}$ & $\langle V-R\rangle_{0}$ & $\langle R-I\rangle_{0}$ & $\log L / L_{\odot}$ & $\log T_{\text {eff }}$ & $\begin{array}{l}\text { Source of } \\
\text { Photometry }\end{array}$ \\
\hline EV Sct & 3.1 & NGC 6664 & -2.9 & 0.29 & 0.47 & $\ldots$ & 0.27 & 3.07 & 3.802 & 1,2 \\
\hline CE Cas b ........... & 4.5 & NGC 7790 & -2.8 & & 0.66 & $\ldots$ & & 3.06 & 3.771 & \\
\hline CE Cas a ........... & 5.1 & NGC 7790 & -2.8 & $\ldots$ & 0.74 & $\ldots$ & $\ldots$ & 3.06 & 3.757 & \\
\hline CV Mon ............ & 5.4 & Anon & -3.0 & $\ldots$ & 0.69 & $\ldots$ & 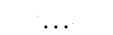 & 3.14 & 3.765 & \\
\hline U Sgr $\ldots \ldots \ldots \ldots$ & 6.7 & M25 & -3.8 & 0.35 & 0.64 & 0.52 & 0.33 & 3.45 & 3.782 & $1,2,3$ \\
\hline DL Cas .............. & 8.0 & NGC 129 & -3.9 & 0.38 & 0.68 & & 0.34 & 3.50 & 3.774 & 1,2 \\
\hline
\end{tabular}

CEPHEIDS IN CLUSTERS

Sources of Photometry.-(1) $\langle b-y\rangle$ from Schmidt 1976 corrected to be consistent with the photometry of Paper I. (2) $\langle R-I\rangle$ from Schmidt 1976. (3) $\langle V-R\rangle$ and $\langle R-I\rangle$ from Schmidt 1971. 


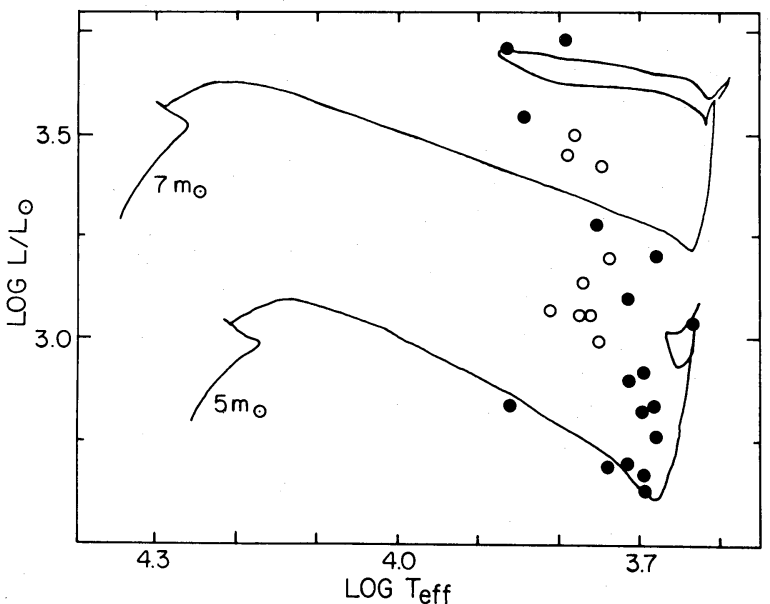

Fig. 1.-The theoretical H-R diagram for the stars in Tables 1 and 2. In this and subsequent diagrams, open circles represent Cepheids, and filled circles represent nonvariables. The evolutionary tracks from Becker (1981) for $(Y, Z)=(0.28,0.02)$ and masses of $5 M_{\odot}$ and $7 M_{\odot}$ are shown.

perature of the Cepheid strip. We should bear this uncertainty in mind in our discussion of these results below.

\section{THEORETICAL INTERPRETATION}

In Figure 1 we have plotted the luminosities of the stars from Tables 1 and 2 against their effective temperatures. Also plotted are evolutionary tracks for stars with $(Y, Z)=(0.28$, 0.02) from Becker (1981). During the phase of core helium burning, stars evolve away from the red giant branch and into the yellow giant region. Such objects will be located in a band near the tips of the blue loops in the evolutionary tracks. Based on the time scales involved, this band should be $0.05-0.10$ wide in $\log T_{\text {eff }}$. It can be seen in Figure 1 that most of our stars occupy a band of about that width near the tips of the blue loops. We are thus justified in identifying these yellow giants with the core helium burning stage of evolution. The following discussion of various questions regarding these stars is based on this assumption.

\section{a) The Edges of the Instability Strip}

The H-R diagram of the yellow giant region is replotted in Figure 2, and it can be seen that the variables and nonvariables bracket the edges of the instability strip and allow us to place limits on their locations. In principle, placing the edges with this data should be more informative than previous determinations which were based on Cepheids only.

Considering first the blue edge of the instability strip, it can be seen that the Cepheids U Sgr and EV Sct are the bluest variables and provide a red limit on the location of the edge. Using theoretical blue edges calculated from the relations given by Iben and Tuggle (1975) with the evolutionary massluminosity relations of Becker, Iben, and Tuggle (1977), we find that EV Sct will lie on the blue edge if we take the helium abundance to be $Y=0.26$ and the metal abundance to be $Z=0.02$ (shown by the solid line in Fig. 2). Since it has been suggested that this star might be an overtone pulsator (Schmidt 1984b), it might be more appropriate to use U Sgr which will lie at the blue edge if $Y=0.23$ and $Z=0.02$ (shown in part by the dot-dash line). These values then provide lower limits on the helium abundance.

The reddest nonvariable star on the blue side of the insta- bility strip is star 2 in NGC 7654. Unfortunately, there is a significant gap between this star and the Cepheids. As the helium abundance is increased, the blue edge becomes hotter until a value of $Y=0.45$ is reached, after which the blue edge moves toward cooler temperatures. At its hottest, the blue edge does not reach the temperature of NGC 7654-2 for $Z=0.02$ (shown in part by the dashed line). If we take $Z=0.01$ and $Y=0.45$, the blue edge nearly reaches this star (shown in part by the dotted line). This is rather extreme and in any event is outside of the range of the model parameters used in obtaining the theoretical blue edges. We conclude that these data provide no upper limit on the helium abundance.

In the preceding paragraph, we assumed that the masses of the Cepheids are evolutionary and that the primordial interior composition (used in the evolutionary calculations) was the same as the envelope composition (used in the pulsation calculations). Both of these assumptions have been questioned. In the study of the luminosities of Cepheids (Schmidt 1984b) we found that the pulsational masses of these stars are lower than the evolutionary masses by a significant amount. The suggestion that helium-enriched envelopes can account for the mass discrepancy of the beat and bump Cepheids (Cox 1980) implies that the primordial interior composition was lower in helium than the envelope. To test these two possibilities, we determined blue edges from the Iben and Tuggle formulae both for the lower masses implied by the pulsation and using interior abundances differing from the envelope. Since the edges of the instability strip are relatively insensitive to the mass, neither

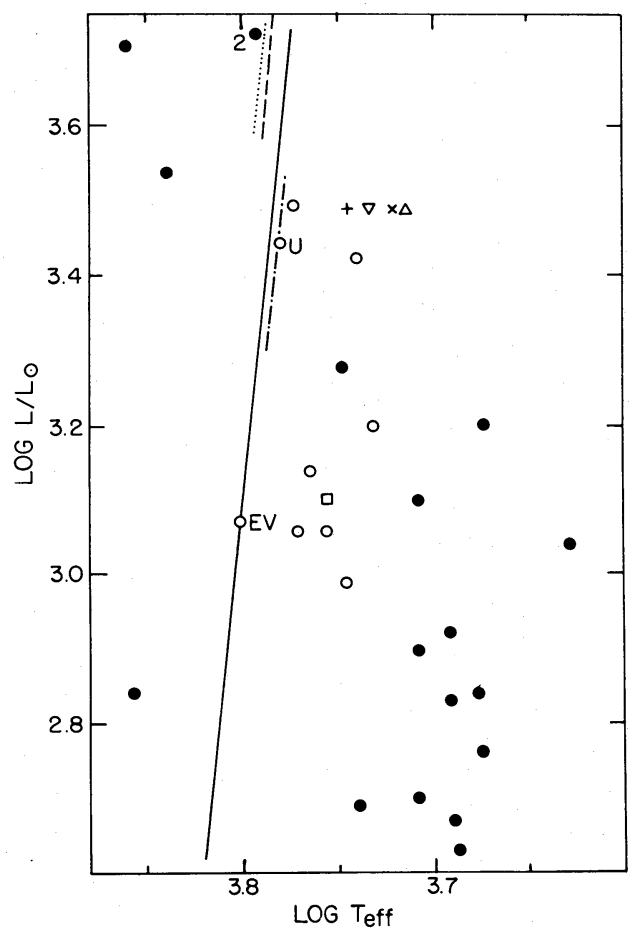

FIG. 2.-The H-R diagram with the edges of the instability strip indicated. The Cepheids U Sgr and EV Sct and the nonvariable NGC 7654-2 are identified. The solid line is the theoretical blue edge for $Y=0.26, Z=0.02$. Portions of the blue edges for $Y=0.23, Z=0.02$ (dot-dash line), $Y=0.45, Z=0.02$ (dashed line), and $Y=0.45, Z=0.01$ (dotted line) are also shown. Theoretical red edge locations are shown with other symbols as follows: $+: Y=0.18$, $Z=0.02$, mass $=6 ; \quad \nabla: Y=0.28, \quad Z=0.02$, mass $=6 ; \quad x: Y=0.35$, $Z=0.02$, mass $=7 ; \triangle: Y=0.28, Z=0.02$, mass $=4 ; \square: Y=0.28, Z=0.02$, mass $=5$. 
change has any appreciable effect on the location of the edges shown in Figure 2. Thus, we are unable to comment on these two possibilities on the basis of the present data.

In addition to the effects of mass and composition, the theoretical blue edges are dependent on such things as the treatment of convection, the radiative opacities, and the atmospheric boundary conditions used in the calculations. These make any conclusions we might draw even less conclusive.

While we are unable to place any interesting limits on the helium abundance using the blue edge or arrive at any conclusions regarding helium-enriched envelopes or pulsational mass discrepancies, we are able to conclude that the theory has satisfactorily predicted the location of the blue edge for a range of reasonable abundances and masses.

The location of the red edge of the instability strip is a difficult theoretical problem, and the only results in the literature are due to hydrodynamic calculations of Deupree (1980). The locations of the red edges for his models are shown in Figure 2 by various symbols. It can be seen that most of the red edges are at temperatures which are too hot compared with the observations and only the most mass deficient model gives a red edge in agreement. The observed width of the strip (850$1400 \mathrm{~K}$ ) is also larger than predicted by Deupree (for fundamental pulsation) unless we adopt a relatively large helium abundance (greater than about $Y=0.34$ ) or a mass smaller than evolutionary values. Given the difficulties of the prediction of the location of the red edge, and the remaining uncertainties in the observational temperatures, it is not surprising that they fail to agree in detail with the observations.

\section{b) Nonvariable Stars within the Cepheid Strip}

It has been suggested that some nonvariable stars are found within the Cepheid instability strip (Fernie and Hube 1971; Schmidt 1972). These suggestions have been based on field stars for which it is difficult to obtain accurate luminosities and colors. Thus, there are uncertainties in the placement of a given star within the instability strip. The present sample of cluster stars does not suffer from this problem.

An examination of Figure 2 shows that only one star, NGC 3114-2 falls among the Cepheids. Both the DDO photometry and the uvby photometry indicate that it is a cluster member. Its location in the sequence of helium core burning stars also supports cluster membership. The reddening in this cluster is small, $E(b-y)=0.06$, and quite uniform so the placement of this star in the H-R diagram is very secure. However, since this is the only example of a nonvariable Cepheid strip star we were able to find in our original sample of 84 stars, efforts to firmly establish its membership should be made; NGC 3114 is an excellent candidate for proper motion or radial velocity studies. Furthermore, star 2 should be checked for variability. For the present we will assume that it is a nonvariable Cepheid strip star.

In Figure 2 we see that there are three nonvariables on the yellow giant branch to the blue of the Cepheid strip in a region approximately equal in width to the strip, while an equally wide area to the red of the Cepheids contains seven stars (excluding the four faintest stars which seem to fall below a continuation of the yellow giant sequence). It is thus reasonable to expect four or five stars within the strip. The discussion of Paper I indicated that the sample considered there was between $40 \%$ and $70 \%$ complete for clusters bright enough to be included. Since great efforts have been made to discover cluster Cepheids, it is not surprising that there are about twice as many Cepheids as the nonvariable sample would predict. In any event, our failure to discover more than a single example of a nonvariable Cepheid strip star (while finding yellow giants on either side of the strip) indicates that these stars are signficantly less common than Cepheids.

Fernie and Hube (1971) and Schmidt, Rosendhal, and Jewsbury (1974) listed a number of bright stars which they contended were within the Cepheid strip. Among the stars on their lists at least four (depending on where the boundaries of the strip are taken to be) are brighter than $V=3$ and nine are brighter than $V=5$. By comparison, there is only one known Cepheid brighter than $V=3$ and only eight brighter than $V=5$. Thus, these studies suggested that nonvariable Cepheid strip stars are at least as numerous as Cepheids. The present result clearly contradicts this. The likely explanation is that the placement of field stars in the H-R diagram is too uncertain to decide whether they lie within the instability strip.

\section{c) The Location of Core Helium Burning Stars}

The band in the H-R diagram occupied by stars during core helium burning is limited on the hot side by the length of the blue loops in the evolutionary tracks. In Figure 3 we have plotted the locations of the blue limits of core helium burning for various compositions from the tabulations of Becker (1981). These were taken from the second of the points labeled B on his tracks. Each of these lines only extends over the range defined by the masses that Becker used for each composition. It can be seen that none of these lines matches the blue envelope of the observed points in both location and slope.

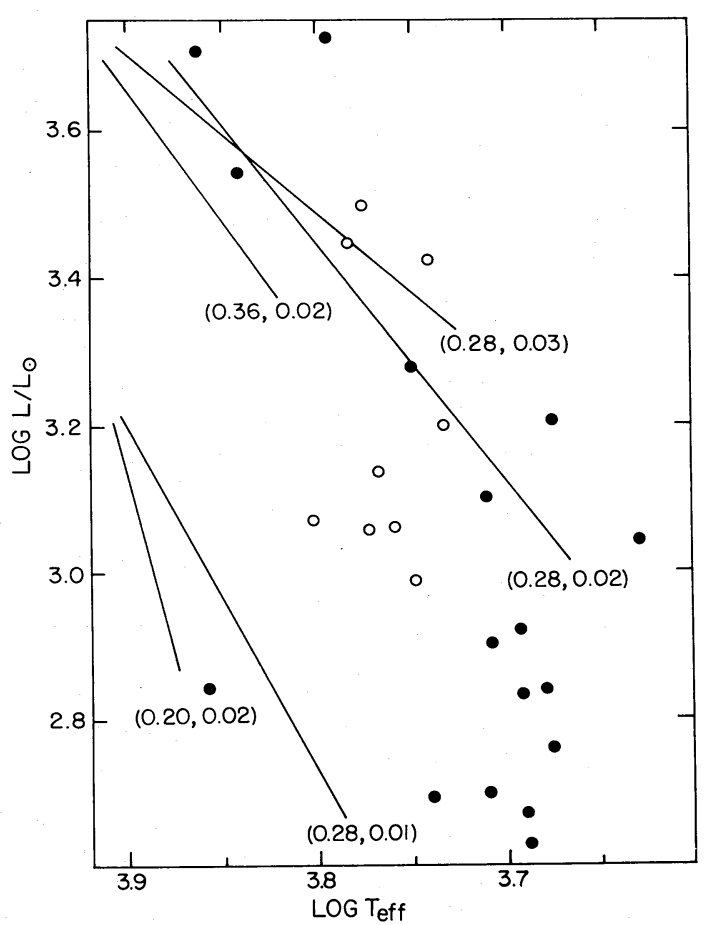

FIG. 3.-The H-R diagram with the blue limits of the core helium burning phase shown. The various lines are for the various compositions used by Becker and are labeled with $Y$ and $Z$. 
To allow us to test the effects of intermediate compositions, we have approximated the blue limit by a linear relation,

$$
\log T_{\text {eff }}=a(\log L-3.5)+b \text {. }
$$

Equations were fitted to values of $a$ and $b$ obtained from the models of Becker for various compositions and the following interpolation formulae resulted:

$$
\begin{aligned}
a= & 0.307+1.6(Y-0.28)-12.5(Y-0.28)^{2} \\
& +14.0(Z-0.02)+530(Z-0.02)^{2}
\end{aligned}
$$

and

$$
\begin{aligned}
b= & 3.815-0.468(Y-0.28)+13.4(Y-0.28)^{2} \\
& -6.35(Z-0.02)+605(Z-0.02)^{2} .
\end{aligned}
$$

Since the model compositions available are limited, these relations must be regarded as very approximate; they are only valid for small departures from $(Y, Z)=(0.28,0.02)$. They are adequate, nonetheless, to see whether a reasonable composition can be found for which equation (3) fits the blue envelope of the points in Figure 3.

The observed points are replotted in Figure 4 where a solid line has been drawn by eye to represent the blue envelope of the observed points. It fits equation (1) with $a=0.157$ and $b=3.833$. No solution to equations (4) and (5) exists for these values. The closest which can be found is for $(Y, Z)=(0.233$, 0.021 ) with $a=0.219$ and $b=3.862$. This is plotted as a dotdash line in Figure 4. It can be seen that is is a reasonable approximation to the blue limit for the observed points. However, this value for $Y$ is unreasonably small. Additionally,

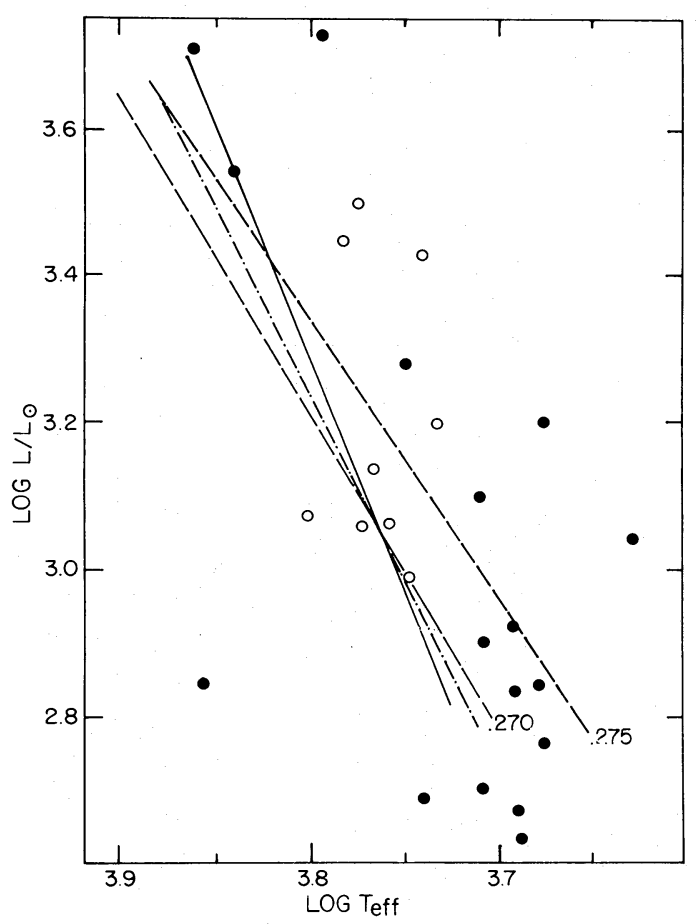

Fig. 4.-The H-R diagram with various blue limits for core helium burning shown. The solid line is an eye-fitted envelope to the observed points. The dot-dash line is the best fit obtained with equation (3). It corresponds to $(Y, Z)=(0.233,0.021)$. The dashed lines are for helium abundances of $Y=0.270$ and $Y=0.275$ and $Z$ from eq. (6).
$Y$ and $Z$ are not independent parameters and should be constrained by a relationship such as

$$
Y=0.228+2.83 Z
$$

which was derived by Lequeux et al. (1979) from observations of $\mathrm{H}$ II regions in various galaxies. If we use equation (6) to relate $Y$ and $Z$, we obtain a family of curves from equations (3), (4), and (5). A couple near the correct location are shown by dashed lines in Figure 4. They are labeled with $Y$. It can be seen that neither of them reproduces both the slope and the location of the blue envelope. Perhaps the best fit is for $(Y, Z)=(0.27$, 0.015).

We conclude that the theoretical curves do not match the observations for any reasonable composition. This is even more true if a steeper blue limit were drawn in Figure 4 to accommodate all the Cepheids and the fainter nonvariables. While the models cover too coarse a grid of composition to allow an accurate comparison, it does not appear likely that the disagreement is solely a result of an admittedly crude interpolation. Rather, it seems probable that the failure of the comparison results from shortcomings in either the theory of the conversion of the observations to theoretical parameters. If the problem is with the temperature scale, then it would require a luminosity-dependent error since the slope of the blue limit is a major source of the problem. Since the colors used for the temperature are thought to be relatively insensitive to luminosity, this seems unlikely. Therefore, it is more attractive to attribute the discrepancy to effects not taken into account in the evolutionary calculations.

\section{d) The Age Spread in Clusters}

There are six clusters in Table 1 which contain more than one yellow giant each. The locations of the yellow giants in these clusters are shown in Figure 5. Also shown are evolutionary tracks from Becker (1981) for a composition of $Y=0.28$ and $Z=0.02$. It can be seen that the locations of the yellow giants for most of the clusters are consistent with the evolutionary tracks on the assumption that the stars in the cluster are coeval.

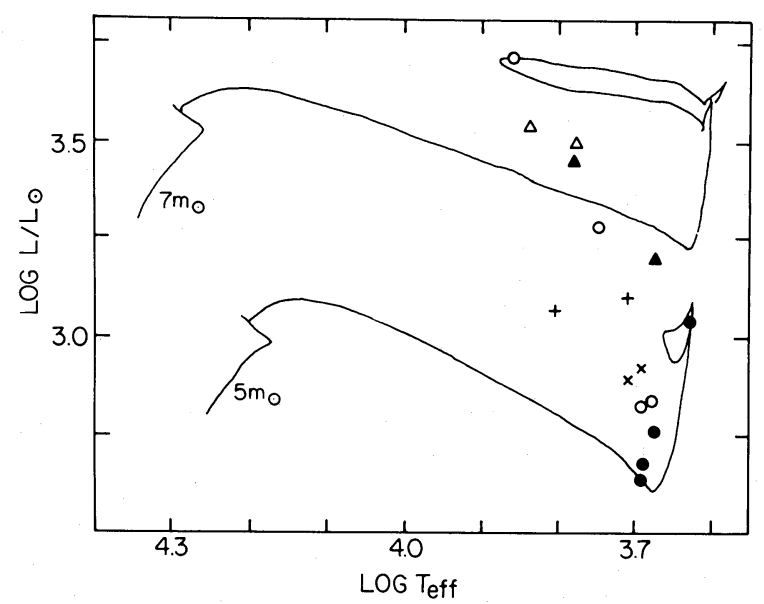

FIG. 5.-The H-R diagram for stars in several clusters denoted by various symbols as follows: solid circles, NGC 2287; open circles, NGC 3114; filled triangles, M25; open triangles, NGC 129; x's, NGC 2546; crosses, NGC 6664. Also shown are the evolutionary tracks from Becker (1981) for $Y=0.28$ and $Z=0.02$. 
In NGC 3114 the yellow giants (open circles in Fig. 5) span a luminosity range of almost $3 \mathrm{mag}$. This cluster by itself defines the sequence of helium core burning stars. In comparing the data for this cluster with the evolutionary tracks, there is ambiguity as to the crossing of the yellow giant region on which a particular star is located. Using the evolutionary tracks and the associated time scales, we can estimate the age of each star for various assumptions regarding the evolutionary stage. If we assume that stars 76 and 159 are on the first crossing, star 2 is on the second crossing, and star 1 is on the third crossing, the discrepancy among the four stars is the smallest. The implied range in age is then 14 million years (from 39 to 52 million years). In fact, based on the time scales of the evolutionary tracks, it is unlikely that this assignment of stars to various crossings is correct. The majority should be on the second crossing or near the blue limit of the core helium burning loop. This results in a range from 39 million years to 87 million years. While the spread (48 million years) is large, these values do bracket the age estimate from four-color photometry of main-sequence stars, viz., 65-75 million years (Schmidt 1982b).

In M25 (filled triangles) it can be seen that there is a singificant difference between the locations of the two stars. This implies a difference in age of 14-16 million years if both are on the second or third crossing. This drops to 7 million years if we assume one is on the second crossing and the other on the third.

The Cepheid EV Sct in NGC 6664 lies at a hotter temperature than either the blue loops of the evolutionary tracks or the sequence of other stars (see, for example, its location in Fig. 2). This could be accounted for by its being on the first crossing. If this is correct, its age is in reasonable agreement with the other star for that cluster, NGC 6664-29, assuming it is also on the first crossing. However, this is unlikely, and placing NGC 6664-29 on the second crossing gives an age spread of 21 million years.

The age spreads in the other clusters implied by the yellow giants are all a few million years or less under reasonable assumptions about the crossing on which particular stars are located. In NGC 2287 the stars are parallel to the red giant branch but are somewhat too hot. It is likely that these stars are in that stage of evolution, but the temperature scale of either the observations or the theoretical calculations is slightly in error.

There are several possible explanations for the spread of stars in the yellow giant region. Since the range in luminosity in NGC 3114 is so large we can eliminate the presence of binaries as the explanation for most of the effect. Other effects on the evolution such as various amounts of mass loss, the effects of rotation, the effects of mixing or magnetic fields are possibilities, but none are amenable to detailed discussion on a theoretical basis at present. We will therefore restrict the present comments to the possibility of a range of age among the giants in a cluster.

Herbst and Miller (1982) derived the age spread in NGC 3293 and summarized data in several clusters (all younger than the present clusters). The age spreads of the clusters they discussed ranged from 10 million to 17 million years except for the Pleiades for which the spread was between 50 and 400 million years. Jakobsen (1981) has also discussed age spread in clusters and derived values between 10 and 100 million years. From these it is obvious that the values we have obtained are quite reasonable, and the scatter of stars in the clusters can be rea- sonably explained in terms of an age spread combined with the multiple crossing of the yellow giant region by the evolutionary tracks.

\section{e) Location of Helium Core Burning Stars Relative to Hydrogen Core Burning Stars}

The evolutionary tracks predict that stars which are crossing the instability strip for the first time after evolving off the main sequence will be less luminous than the brightest stars at the turnoff point. These stars then evolve back into the yellow giant region at somewhat higher luminosity and, depending on the composition, are slightly more or less luminous than stars at the turnoff point. This prediction of the theory can be compared directly to the stars in our sample.

We used the four-color and $\mathrm{H} \beta$ photometry for each cluster (omitting NGC 2546 for lack of such data) to select the most luminous main-sequence stars. Stars which appeared to be blue stragglers were ignored as were those which appeared to be nonmembers on the basis of the four-color photometry. In practice, there were often several stars near the top of the main sequence, and the results were not sensitive to which was chosen. To obtain the luminosities of these stars we have used the bolometric corrections of Code et al. (1976). These bolometric corrections were in terms of $(B-V)$ but were transformed to $c_{1}$. The use of $c_{1}$ is superior to $B-V$ because it is more sensitive to temperature for B stars and is less sensitive to interstellar absorption. For each star in Tables 1 and 2 we determined the difference in luminosity between the turnoff point and the yellow giants. These are plotted in Figure 6 against the yellow giant luminosity.

To obtain the theoretical luminosity difference, the most populated portion of the yellow loops of the evolutionary tracks of Becker (1981) was located using the evolutionary times he tabulated. The end of the main sequence was taken to be Becker's first point labeled B. The luminosity difference depends on composition, and we have plotted the limits of the yellow giant region for two choices. $(Y, Z)=(0.28,0.02)$ was plotted because it is the most likely Population I composition, while $(Y, Z)=(0.28,0.01)$ gave the largest luminosity difference

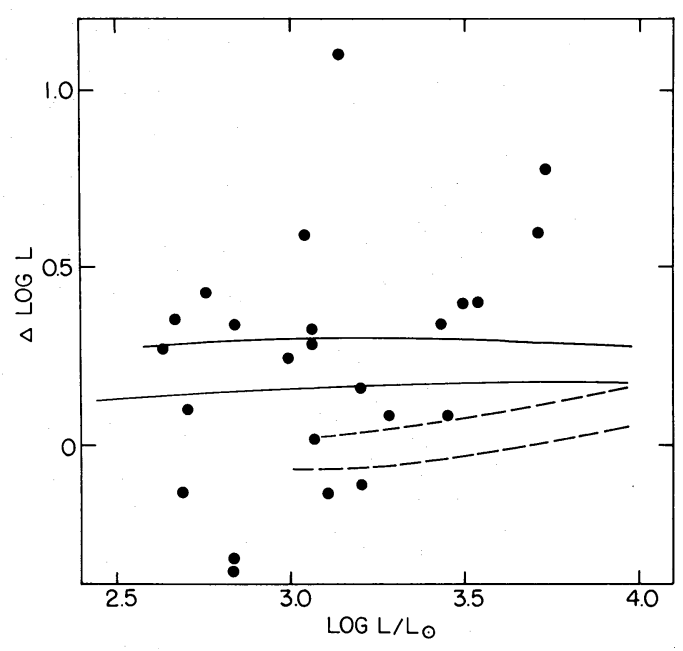

FIG. 6.-The luminosity difference between the yellow giants and the most luminous main-sequence stars plotted atainst the yellow giant luminosity. The theoretical ranges are shown for $(Y, Z)=(0.28,0.02)$ (dashed curves) and $(Y, Z)=(0.28,0.01)$ (solid curves) 
for any of Becker's compositions. It can be seen in Figure 6 that while the theoretical band for $Z=0.01$ lies near the center of the observed distribution, the scatter is much larger than the theory can explain. Several possible explanations will be considered.

The apparent magnitudes of either the main-sequence stars or the yellow giants may be affected significantly by companions. However, this explanation is unlikely on several grounds. In the first place, it cannot explain stars which fall more than a factor of 2 out of the proper luminosity range. There are four such objects in Figure 6. The study of Abt and Levy (1978) indicated that between $11 \%$ and $16 \%$ of early B stars have companions with masses at least half as large as the primary. Assuming that these statistics apply approximately to the late B stars in the clusters and to the B star precursors of the Cepheids, we estimate that about three of our yellow giants and three of the stars at the top of the main sequence will have such companions. A companion of half the mass of either the yellow giant or the main sequence star will increase $\log L$ by only 0.03 . Thus, we should expect only about six stars outside of the theoretical band in Figure 6 by more than that amount. There are 3 times that number. Thus, it appears that simple contamination of the light of either the brightest mainsequence stars or the yellow giants by companions cannot explain the scatter in Figure 6.

If there are gaps in the luminosity functions in some clusters, it will introduce scatter in the luminosity differences. This will not explain the stars which scatter below the theoretical band, however, and it is unlikely to be the explanation for the scatter in Figure 6.

The scatter in Figure 6 might possibly be explained by effects which were not included in the theoretical calculations but which affect the main-sequence evolution. Since we observe a scatter, the cause must operate in a stochastic manner. Becker and Mathews (1983) suggested that convective overshoot might cause the discrepancies they found in comparing theoretical H-R diagrams with observation in NGC 1866. To this we might add rotation, mass loss, and interacting binaries as further possibilities to be considered. While it is beyond the scope of this paper to pursue these possibilities, these data do suggest the importance to post-main-sequence evolution of processes which were omitted from the models. Additionally, arguments concerning the membership of Cepheids or other evolved stars in clusters based on their location compared to the main sequence must be viewed with caution (e.g., Flower 1978).

\section{SUMMARY}

The yellow giants and Cepheids place limits on the location of the edges of the instability strip. There is no disagreement with theory for reasonable compositions, but these data do not place any interesting constraints on the model parameters. There is only one nonvariable star in our sample within the instability strip. This is less than expected on the basis of previous studies of nonvariable Cepheid strip stars and implies that some field stars have been placed in the strip through errors in intrinsic colors or luminosities. The distribution of these stars in the H-R diagram agrees in appearance with what is expected for helium core burning stars. However, a detailed comparison with the evolutionary model calculations shows that the exact location is not reproduced with any reasonable composition. For those clusters with more than one yellow giant, we have compared the locations of the evolved stars with the evolutionary tracks. There is good agreement with the assumption that most of the stars are in the second or third crossing of the yellow giant region, although several clusters require age spreads of between 20 and 50 million years. A comparison is made of the most luminous early-type stars and the yellow giants. Although the luminosity difference agrees roughly with theory on the average, there is considerable scatter which cannot be accounted for in terms of observational errors or the presence of binary stars.

It appears that while the theory of stellar evolution agrees with the main features of the observations of yellow giants, there are discrepancies when detailed comparisons are made. It is likely that these arise from phenomena which are neglected in the theoretical calculations. At least some of these effects seem to introduce scatter and are thus different from star to star or at least from cluster to cluster.

This research was supported by the National Science Foundation through grant AST 83-07665. The data used were obtained at Kitt Peak National Observatory and at Cerro Tololo Inter-American Observatory. The use of these facilities is gratefully acknowledged.

\section{REFERENCES}

Abt, H. A., and Levy, S. G. 1978, Ap. J. Suppl., 36, 241.

Becker, S. A. 1981, Ap. J. Suppl., 45, 475.

Becker, S. A. Iben, I, and Tuggle, R. S. 1977, Ap. J., 218, 633.

Becker, S. A., and Mathews, G. J. 1983, Ap. J., 270, 155.

Becker, W., and Fenkhart, R. 1971, Astr. Ap. Suppl., 4, 241.

Code, A. D., Davis, J., Bless, R. C., and Hanbury Brown, R. 1976, Ap. J., 203

417

Cox, A. N. 1979, Ap. J., 229, 212

1980, Ann. Rev. Astr. Ap., 18, 15

Crawford, D. L. 1978, A.J., 83, 48 .

Crawford, D. L., and Mandewewala, N. 1976, Pub. A.S.P., 88, 917

Danford, S. C., and Thomas, J. 1981, Pub. A.S.P.,93, 447.

Deupree, R. G. 1980, Ap. J., 236, 225.

Eggen, O. J. 1981, Ap. J., 247, 507. $1983 a$, A.J., 88, 379 $1983 b, A . J, \mathbf{8 8}, 386$

Fernie, J. D. A.J., 68, 780 .

Fernie, J. D., and Hube, J. 1971, Ap. J., 168, 437

Flower, P. J. 1978, $A p$. J., 224, 948

Forbes, D., and Schmidt, E. G. 1984, in preparation

Herbst, W., and Miller, D. P. 1982, A.J., 87, 1478 .

Iben, I., and Tuggle, R. S. 1975, Ap. J., 197, 39.
Jakobsen, A. M. 1981, Pub. A.S.P., 93, 547.

Johnson, H. L. 1966, Ann. Rev. Astr. Ap., 4, 193.

Lequeux, S., Peimbert, M., Rayo, J. F., Serrano, A., and Torres-Peimbert, S. 1979, Astr. Ap., 80, 155

Pel, J. W. 1978, Astr. Ap., 62, 75 .

1980, in Current Problems in Stellar Pulsational Instabilities, ed. D. Fischel, J. R. Lesh, and W. M. Sparks (NASA TM 80625), p. 1

Sandage, A. R., and Gratton, L. 1963, in Star Evolution, International School of Physics "Enrico Fermi," Vol. 28, ed. L. Gratton (New York: Academic Press), p. 11.

Schmidt, E. G. 1971, Ap. J., 165, 335

- 1972, Ap. J., 172, 679.

1976, Ap.J., 203, 466

1980, A.J., 85, 695

1982a, Pub. A.S.P., 94, 232

$1982 b$, A.J., 87, 650

$1982 c$, A.J., 87, 1197.

1984a, Ap. J. Suppl., 55, 455 (Paper I).

$1984 b$, Ap. J., 285, 000

Schmidt, E. G., Rosendhal, J. D., and Jewsbury, C. P. 1974, Ap. J., 189, 293.

Snowden, M. S. 1976, Pub. A.S.P., 88, 179. 